Korean J. Math. 20 (2012), No. 2, pp. 185-191

\title{
INTEGRAL DOMAINS WITH FINITELY MANY STAR OPERATIONS OF FINITE TYPE
}

\author{
Gyu Whan Chang
}

\begin{abstract}
Let $D$ be an integral domain and $S F(D)$ be the set of star operations of finite type on $D$. We show that if $|S F(D)|<\infty$, then every maximal ideal of $D$ is a $t$-ideal. We give an example of integrally closed quasi-local domains $D$ in which the maximal ideal is divisorial (so a $t$-ideal) but $|S F(D)|=\infty$. We also study the integrally closed domains $D$ with $|S F(D)| \leq 2$.
\end{abstract}

\section{Introduction}

Let $D$ be an integral domain with quotient field $K$. Let $\mathbf{F}(D)$ be the set of nonzero fractional ideals of $D$. A mapping $I \mapsto I^{*}$ of $\mathbf{F}(D)$ into $\mathbf{F}(D)$ is called a star-operation on $D$ if for all $0 \neq a \in K$ and $I, J \in \mathbf{F}(D)$, the following conditions are satisfied:

(1) $(a D)^{*}=a D$ and $(a I)^{*}=a I^{*}$,

(2) $I \subseteq I^{*} ; I \subseteq J$ implies $I^{*} \subseteq J^{*}$, and

(3) $\left(I^{*}\right)^{*}=I^{*}$.

Given any star operation $*$ on $D$, one can construct a new star operation $*_{f}$ by setting $I^{*}=\cup\left\{J^{*} \mid J\right.$ is a nonzero finitely generated subideal of $I\}$ for all $I \in \mathbf{F}(D)$. A star operation $*$ on $D$ is said to be of finite type if $*_{f}=*$. Obviously, $\left(*_{f}\right)_{f}=*_{f}$, and hence $*_{f}$ is of finite type. Clearly, $I^{*}=I^{*_{f}}$ for all nonzero finitely generated fractional ideals $I$ of $D$; so if $D$ is a Noetherian domain, then each star operation on $D$ is of finite type. An $I \in \mathbf{F}(D)$ is called a $*$-ideal if $I^{*}=I$, while a $*$-ideal is called

Received April 7, 2012. Revised June 7, 2012. Accepted June 10, 2012.

2010 Mathematics Subject Classification: 13A15, 13 G05.

Key words and phrases: star operation of finite type, Prüfer domain, pseudovaluation domain.

This work was supported by Basic Science Research Program through the National Research Foundation of Korea (NRF) funded by the Ministry of Education, Science and Technology (2010-0007069). 
a maximal $*$-ideal if it is maximal among proper integral $*$-ideals. Let $*$ $\operatorname{Max}(D)$ denote the set of maximal $*$-ideals of $D$. It is well known that a maximal $*$-ideal is a prime ideal; each prime ideal minimal over a $*_{f}$-ideal is a $*_{f}$-ideal; and $*_{f}$-Max $(D) \neq \emptyset$ if $D$ is not a field. A star operation * on $D$ is said to be stable if $(I \cap J)^{*}=I^{*} \cap J^{*}$ for all $I, J \in \mathbf{F}(D)$. Recall that $*$ is endlich arithmetisch brauchbar (e.a.b.) if $(A B)^{*} \subseteq(A C)^{*}$ for all nonzero finitely generated fractional ideals $A, B, C$ of $D$ implies $B^{*} \subseteq C^{*}$.

The most well-known examples of star operations are the $d-, v-$, and $t$-operations. The $d$-operation is just the identity function on $\mathbf{F}(D)$; so $d=d_{f}$. The $v$-operation is defined by $I_{v}=\left(I^{-1}\right)^{-1}$, where $I^{-1}=\{x \in$ $K \mid x I \subseteq D\}$, and the $t$-operation is given by $t=v_{f}$. We say that a $v$-ideal is a divisorial ideal. For two star operations $*_{1}$ and $*_{2}$ on $D$, we mean by $*_{1} \leq *_{2}$ that $I^{*_{1}} \subseteq I^{*_{2}}$ for all $I \in \mathbf{F}(D)$. Clearly, if $*_{1} \leq *_{2}$, then $\left(*_{1}\right)_{f} \leq\left(*_{2}\right)_{f}$. We know that if $*$ is any star operation on $D$, then $d \leq * \leq v$, and hence $d \leq *_{f} \leq t$. For basic properties of star operations, see [7, Sections 32 and 34].

Let $S(D)$ (resp., $S F(D)$ ) be the set of star operations (resp., star operations of finite type) on $D$; so $S F(D) \subseteq S(D)$. In [11, Proposition 2.1], it was shown that if $|S(D)|<\infty$, then each maximal ideal of $D$ is a $t$-ideal. It is clear that if $|S(D)|<\infty$, then $|S F(D)|<\infty$, but not vice versa (for example, if $D$ is an h-local Prüfer domain that has infinitely many nondivisorial maximal ideals, then $|S F(D)|=1$ and $|S(D)|=\infty[11$, Corollary 3.2]). So it is reasonable to ask what happens if $|S F(D)|<\infty$. Specifically, is it true that $|S F(D)|<\infty$ if and only if each maximal ideal of $D$ is a $t$-ideal ? The purpose of this paper is to give an answer to this question. Precisely, we show that if $|S F(D)|<\infty$, then each maximal ideal of $D$ is a $t$-ideal. We give an example of integrally closed domains $D$ in which each maximal ideal is a $t$-ideal but $|S F(D)|=\infty$. We also study the integrally closed domains $D$ with $|S F(D)| \leq 2$.

\section{Main Results}

Let $D$ be an integral domain with quotient field $K$. Let $S(D)$ (resp., $S F(D)$ ) be the set of star operations (resp., star operations of finite type) on $D$. 
We begin this section with a necessary condition for $|S F(D)|<\infty$, which is a simple modification of [11, Proposition 2.1(2)] that if $|S(D)|<$ $\infty$, then each maximal ideal of $D$ is a $t$-ideal.

Lemma 1. Let $I$ be a nonzero finitely generated ideal of $D$ with $I_{v}=D$. For each integer $n \geq 1$, let $E^{*_{n}}=\left(I^{n}:\left(I^{n}: E\right)\right)$ for all $E \in \mathbf{F}(D)$. Then $*_{n}$ is a star operation on $D$ such that $\left(*_{n}\right)_{f} \neq\left(*_{m}\right)_{f}$ for all positive integers $n \neq m$.

Proof. Note that $\left(I^{n}: I^{n}\right)=D$; so $*_{n}$ is a star operation on $D$ [10, Proposition 3.2]. Also, by the proof of [11, Proposition 2.1], for $0<m<n,\left(I^{n}\right)^{*_{n}}=I^{n}$ and $\left(I^{n}\right)^{*_{m}}=I^{m}$. Note that $I^{n} \neq I^{m}$ for $n \neq m\left[13\right.$, Theorem 76] and $I^{n}$ is finitely generted for all $n \geq 1$. Hence $\left(I^{n}\right)^{\left(*_{n}\right)_{f}}=\left(I^{n}\right)^{*_{n}}=I^{n} \neq I^{m}=\left(I^{n}\right)^{*_{m}}=\left(I^{n}\right)^{\left(*_{m}\right)_{f}}$. Thus $\left(*_{n}\right)_{f} \neq$ $\left(*_{m}\right)_{f}$.

Theorem 2. If $|S F(D)|<\infty$, then each maximal ideal of $D$ is a $t$-ideal.

Proof. Assume to the contrary that there is a maximal ideal $M$ of $D$ with $M_{t}=D$. Then there is a nonzero finitely generated subideal $I$ of $M$ such that $I_{v}=I_{t}=D$. Hence if we set $E^{*_{n}}=\left(I^{n}:\left(I^{n}: E\right)\right)$ for each $E \in \mathbf{F}(D)$, then $*_{n}$ is a star operation on $D$ such that $\left(*_{n}\right)_{f} \neq\left(*_{m}\right)_{f}$ for all positive integers $m \neq n$ by Lemma 1 . Thus $|S F(D)|=\infty$, a contradiction. Thus each maximal ideal of $D$ is a $t$-ideal.

Let $S F_{s}(D)$ be the set of stable star operations of finite type on $D$; so $S F_{s}(D) \subseteq S F(D)$. In [3, Theorem 4], it was shown that if $\Omega$ is the set of nonzero prime ideals $P$ of $D$ with $P_{t}=D$, then $|\Omega|+1 \leq\left|S F_{s}(D)\right| \leq 2^{|\Omega|}$. Hence each maximal ideal of $D$ is a $t$-ideal if and only if $\left|S F_{s}(D)\right|=1$.

Corollary 3. If $\left|S F_{s}(D)\right| \geq 2$, then $|S F(D)|=\infty$.

Proof. If $\left|S F_{s}(D)\right| \geq 2$, then $D$ has at least one maximal ideal that is not a $t$-ideal $[3$, Theorem 4]. Thus $|S F(D)|=\infty$ by Theorem 2 .

As in [8], we say that a prime ideal $P$ of $D$ is strongly prime if $x y \in P$ and $x, y \in K$ imply $x \in P$ or $y \in P$, while $D$ is a pseudo-valuation domain (PVD) if every prime ideal of $D$ is strongly prime. It is known that $D$ is a PVD if and only if $D$ is quasi-local whose maximal ideal is strongly prime if and only if there exists a valuation overring $V$ of $D$ such that $\operatorname{Spec}(V)=\operatorname{Spec}(D)$ [8, Theorem 2.7]. 
We next give an example of integral domains whose maximal ideals are $t$-ideals but $|S F(D)|=\infty$, which shows that the converse of Theorem 2 does not hold.

EXAMPLE 4 . Let $\mathbb{R}$ be the field of real numbers, $y, z$ be indeterminates over $\mathbb{R}, K=\mathbb{R}(y, z)$ be the quotient field of the polynomial ring $\mathbb{R}[y, z]$, $X$ be an indeterminate over $K, V=K \llbracket X \rrbracket$ be the power series ring over $K$ (so $V$ is a rank-one DVR), and $D=\mathbb{R}+X K \llbracket X \rrbracket$. It is clear that $D$ is an integrally closed PVD, $V / X K \llbracket X \rrbracket=K$, and $D / X K \llbracket X \rrbracket=\mathbb{R}$ (so $\operatorname{trdeg}(K, \mathbb{R})=2$ ). Hence $D$ has infinitely many e.a.b. star operations of finite type [4, Theorem 4.10]. Thus $|S F(D)|=\infty$.

Given an e.a.b. star operation on an integrally closed domain $D$, the Kronecker function ring of $D$ with respect to $*$ is defined by

$$
K r(D, *)=\{0\} \cup\left\{\frac{f}{g} \mid 0 \neq f, g \in D[X] \text { and } c(f)^{*} \subseteq c(g)^{*}\right\},
$$

where $c(h)$ denotes the ideal of $D$ generated by the coefficients of an $h \in D[X]$. It is well known that $K r(D, *)$ is a Bezout domain and $K r(D, *) \cap K=D[7$, Theorem 32.7].

Let $S F_{e}(D)$ be the set of e.a.b. star operations of finite type on $D$. It is known that $S F_{e}(D) \neq \emptyset$ if and only if $D$ is integrally closed [7, Corollary 32.8]. Also, there is a bijection between $S F_{e}(D)$ and the set of Kronecker function rings of $D$ (cf. [7, Remark 32.9]). We next give a lower bound of $\left|S F_{e}(D)\right|$. (Note that Example 4 shows that the equality of Proposition 5 need not hold, but the equality attains when $D$ is a Prüfer domain.)

Proposition 5. If $D$ is integrally closed, then $\left|S F_{s}(D)\right| \leq\left|S F_{e}(D)\right|$.

Proof. Let $* \in S F_{s}(D)$. Then we can construct an e.a.b. star operation $*_{c}$ of finite type on $D$ such that $*-\operatorname{Max}(D)=*_{c}-\operatorname{Max}(D)[2$, Lemma 3.1]. Recall that if $*^{\prime} \in S F_{s}(D)$, then $I^{*^{\prime}}=\cap_{P \in *^{\prime}-\operatorname{Max}(D)} I D_{P}$ for all $I \in \mathbf{F}(D)$ [1, Corollary 4.2]; so if $*_{1} \in S F_{s}(D)$ with $*_{1} \neq *$, then $*_{1}-\operatorname{Max}(D) \neq *-\operatorname{Max}(D)$. Hence $*_{c}-\operatorname{Max}(D) \neq\left(*_{1}\right)_{c}-\operatorname{Max}(D)$, and thus $*_{c} \neq\left(*_{1}\right)_{c}$. This completes the proof.

We next study the integrally closed domains $D$ with $|S F(D)| \leq 2$. To do this, we first need the notion of a $b$-operation that is an e.a.b. star operation of finite type on an integrally closed domain $D$ defined by $E^{b}=\cap\{E V \mid V$ is a valuation overring of $D\}$ for all $E \in \mathbf{F}(D)[7$, pp. 397-398]. Clearly, the $b$-operation is defined on $D$ if and only if $D$ is 
integrally closed [7, Corollary 32.8]. Also, it is easy to see that $d=b$ if and only if $D$ is a Prüfer domain [7, Theorem 24.7]. This result implies the following theorem.

TheOREm 6. If $D$ is integrally closed, the following statements are equivalent.

(1) $D$ is a Prüfer domain.

(2) $|S F(D)|=1$.

(3) $\left|S F_{s}(D)\right|=|S F(D)|<\infty$.

Proof. (1) $\Rightarrow$ (3) If $D$ is a Prüfer domain, then $d=t$, and thus $S F_{s}(D)=S F(D)=\{d\}$. (3) $\Rightarrow(2)$ This follows directly from Corollary 3. $(2) \Rightarrow(1)$ Note that $\{d, b\} \subseteq S F(D)$; so $d=b$. Thus $D$ is a Prüfer domain.

Recall that $D$ is a $v$-domain if the $v$-operation on $D$ is an e.a.b star operation; so $\operatorname{Kr}(D, v)$ is defined on a $v$-domain $D$ and $\operatorname{Kr}(D, b) \subseteq$ $K r(D, *) \subseteq K r(D, v)$ for any e.a.b. star operation $*$ on $D$. It is known that $D$ is a $v$-domain if and only if each nonzero finitely generated ideal of $D$ is $v$-invertible [7, Theorem 34.6]. Also, $b=t$ if and only if $D$ is a $v$-domain [5, Proposition 35]. As in [4], we say that $D$ is a vacant domain if $D$ has a unique Kronecker function ring. It is clear that $D$ is a vacant domain if and only if the $b$-operation is a unique e.a.b. star operation of finite type on $D$.

It is clear that $\mathrm{P} v \mathrm{MDs}$ are $v$-domains, but $v$-domains need not be $\mathrm{P} v \mathrm{MDs}$ (for example, a one-dimensional completely integrally closed domain that is not a valuation domain is a $v$-domain but not a PvMD (cf. [6, pp. 157-161])). However, if each maximal $t$-ideal of $D$ is divisorial, then $v$-domains are $\mathrm{P} v \mathrm{MDs}$. (For if $I$ is a nonzero finitely generated fractional ideal of $D$, then $\left(I I^{-1}\right)_{v}=D$, and hence $I I^{-1} \nsubseteq P$, because $P_{v}=P$, for all $P \in t-\operatorname{Max}(D)$. Thus $\left(I I^{-1}\right)_{t}=D$.)

THEOREM 7. If $D$ is an integrally closed domain with $|S F(D)|=2$, then

(1) $D$ is not a Prüfer domain,

(2) $D$ is a vacant $v$-domain whose maximal ideals are $t$-ideals, and

(3) $D$ has a nondivisorial maximal $t$-ideal.

Proof. (1) This follows directly from Theorem 6 .

(2) Recall that $d \leq b \leq t$. If $d=b$, then $D$ is a Prüfer domain, a contradiction. Hence $b=t$ by hypothesis, and thus $D$ is a vacant 
$v$-domain [5, Proposition 35]. Also, by Theorem 2, each maximal ideal of $D$ is a $t$-ideal.

(3) By the remark before Theorem 7, if each maximal ideal of $D$ is divisorial, then a $v$-domain is a Prüfer domain. Thus $D$ has at least one maximal $t$-ideal that is not a divisorial ideal.

Corollary 8. Let $D$ be an integrally closed PVD with maximal ideal $M$.

(1) If $D$ is not a valuation domain, then $|S F(D)| \geq 3$.

(2) If $|S(D)|=2$, then $D$ is a valuation domain and $M_{v}=D$.

(3) $|S F(D)| \neq 2$.

Proof. (1) If $|S F(D)|=1$, then $D$ is a Prüfer domain by Theorem 6, and since $D$ is quasi-local, $D$ is a valuation domain. Next, if $|S F(D)|=$ 2 , then $D$ is a $v$-domain by Theorem 7 , and hence $D$ is a Prüfer domain because $M_{v}=M$. Thus $D$ is a valuation domain.

(2) Note that $d \leq b \leq t \leq v$; so $d \neq v$ and either $d=b$ or $b=v$. If $d=b$, then $D$ is a valuation domain, and since $d \neq v$, we have $M_{v}=D$ [9, Lemma 5.2]. Next, if $b=v($ so $t=v)$, then $S(D)=S F(D)=\{d, v\}$, and hence $D$ is a valuation domain by (1). But, in this case, $d=t=v$, a contradiction. Moreover, since $d \neq v$, we have $M_{v}=D[9$, Lemma $5.2]$.

(3) If $D$ is a valuation domain, then $|S F(D)|=1$. Thus $|S F(D)| \neq 2$ by $(1)$.

Added to the proof. Recently, Houston, Mimouni and Park showed that if $D$ is an integrally closed domain, then $|S F(D)|<\infty$ if and only if $D$ is a Prüfer domain [12, Theorem 5.3]. Thus, there does not exist an integrally closed domain $D$ with $|S F(D)|=2$ (cf. Theorem 7) and if $D$ is an integrally closed PVD that is not a valuation domain, then $|S F(D)|=\infty$ (cf. Corollary 8(1) and (3)).

\section{References}

[1] D.D. Anderson, Star-operations induced by overrings, Comm. Algebra 16 (1988), 2535-2553.

[2] G.W. Chang, Prüfer *-multiplication domains, Nagata rings, and Kronecker function rings, J. Algebra 319 (2008), 309-319.

[3] G.W. Chang, On the cardinality of stable star operations of finite type on an integral domain, C.R. Math. Acad. Sci. Paris, Ser. I(2012), in press. 
[4] A. Fabbri, Integral domains having a unique Kronecker function ring, J. Pure Appl. Algebra 215 (2011), 1069-1084.

[5] M. Fontana and G. Picozza, On some classes of integral domains defined by Krull's a.b. operations, J. Algebra 341 (2011), 179-197.

[6] M. Fontana and M. Zafrullah, On v-domains: a survey, in: M. Fontana, S. Kabbaj, B. Olberding, I. Swansm (Eds.), Commutative Algebra, Noetherian and Non-Noetherian Persepectives, Springer, New York, 2011, 145-180.

[7] R. Gilmer, Multiplicative Ideal Theory, Queen's Papers in Pure Appl. Math. 90, Queen's University, Kingston, Ontario, 1992.

[8] J.R. Hedstrom and E.G. Housotn, Pseudo-valuation domains, Pacific J. Math. 75 (1978), 137-147.

[9] W.J. Heinzer, Integral domains in which each non-zero ideal is divisorial, Matematika 15 (1968), 164170.

[10] W. Heinzer, J.A. Huckaba, and I. Papick, $m$-canonical ideals in integral domains, Comm. Algebra 26 (1998), 3021-3043.

[11] E. Houston, A. Mimouni, and M.H. Park, Integral domains which admit at most two star operations, Comm. Algebra 39 (2011), 1907-1921.

[12] E. Houston, A. Mimouni, and M.H. Park, Integrally closed domains with only finitely many star operations, preprint.

[13] I. Kaplansky, Commutative Rings, Polygonal Publishing House, Washington, New Jersey, 1994.

Department of Mathematics

University of Incheon

Incheon 406-772, Korea

E-mail: whan@incheon.ac.kr 